\title{
The representations of the arithmetic operations include functional relationships
}

\author{
JAMES A. DIXON \\ College of William and Mary, Williamsburg, Virginia \\ JULIE K. DEETS \\ Trinity University, San Antonio, Texas \\ and \\ ASHLEY BANGERT \\ College of William and Mary, Williamsburg, Virginia
}

\begin{abstract}
Current theories of mathematical problem solving propose that people select a mathematical operation as the solution to a problem on the basis of a structure mapping between their problem representation and the representation of the mathematical operations. The structure-mapping hypothesis requires that the problem and the mathematical representations contain analogous relations. Past research has demonstrated that the problem representation consists of functional relationships, or principles. The present study tested whether people represent analogous principles for each arithmetic operation (i.e., addition, subtraction, multiplication, and division). For each operation, college (Experiments 1 and 2) and 8th grade (Experiment 2) participants were asked to rate the degree to which a set of completed problems was a good attempt at the operation. The pattern of presented answers either violated one of four principles or did not violate any principles. The distance of the presented answers from the correct answers was independently manipulated. Consistent with the hypothesis that people represent the principles, (1) violations of the principles were rated as poorer attempts at the operation, (2) operations that are learned first (e.g., addition) had more extensive principle representations than did operations learned later (multiplication), and (3) principles that are more frequently in evidence developed more quickly. In Experiment 3, college participants rated the degree to which statements were indicative of each operation. The statements were either consistent or inconsistent with one of two principles. The participants' ratings showed that operations with longer developmental histories had strong principle representations. The implications for a structure-mapping approach to mathematical problem solving are discussed.
\end{abstract}

The ability to apply mathematics to real-world domains is an important skill and an important issue for theories of problem solving. How children and adults generate mathematical solutions to novel problems is not well understood. Problems, of course, do not arrive labeled, and although cue words embedded in the problem may help, people apply mathematics to problems that have unique phrasing (Geary, 1994). One important factor in generating mathematical solutions is the person's conceptual or intuitive representation of the problem. The intuitive representation of the problem is a qualitative, nonformal representation of the relationships between the objects in the problem (e.g., Moore, Dixon, \& Haines,

Preparation of this article was supported in part by National Science Foundation Grant BCS-9996353. We are grateful to Jennifer Abernathy and Justin Green for help with data collection and to Jamie Campbell and David Geary for comments on an earlier version of the article. We also thank the participating schools and students. Correspondence concerning this article should be addressed to J. A. Dixon, Department of Psychology, College of William and Mary, P. O. Box 8795, Williamsburg, VA 23187-8795 (e-mail: jadixo@wm.edu).
1991). Three major lines of research have demonstrated a strong relationship between people's intuitive representations and their mathematical solutions. First, research on comprehending word problems has shown that how people interpret and represent the problem affects their solution strategies (see, e.g., Kintsch \& Greeno, 1985). Second, research comparing experts and novices has shown that the problem representation of experts is superior to that of novices (e.g., Schneider, Gruber, Gold, \& Opwis, 1993). Differences in the problem representation explain many of the differences in the formal solutions used to solve the problem (e.g., Hardiman, Dufresne, \& Mestre, 1989). Third, research on analogy has shown that being able to analogically map the structure of a new problem to a better understood problem improves performance with formal strategies (e.g., Novick \& Holyoak, 1991; Ross \& Kilbane, 1997).

Although a person's intuitive representation of the problem is strongly related to the mathematical solution he or she generates, past work has shown that the relationship is not simple. Having a good intuitive representation of a problem is necessary, but not sufficient, to arrive 
at an appropriate mathematical solution (Dixon \& Moore, 1996, 1997). One implication of this relationship is that the intuitive representation does not automatically cue the appropriate mathematics. That is, knowing how a physical system works (e.g., how temperatures combine) does not give direct access to the mathematics that instantiates those relationships. Rather, intuitive representations and mathematical solutions appear to be related through a more complex process. Dixon and Moore $(1996,1997)$ and Bassok, Chase, and Martin (1998) have independently proposed that this process is similar to structure mapping in analogy (Gentner, 1983; Gentner \& Markman, 1997). Under this hypothesis, people select a mathematical operation on the basis of its structural match to their intuitive representation.

A central but untested prediction of this hypothesis is that people represent relational information about each mathematical operation that is analogous to the relational information in their intuitive representation. That is, just as the intuitive representation contains information about the relationships between objects in the physical system, the representation of each mathematical operation should contain information about the relationships between objects in the mathematical system (i.e., operands and answers). The purpose of this article is to propose and provide evidence for such a representation of the arithmetic operations of addition, subtraction, multiplication, and division.

\section{Principle Representation of Mathematical Operations}

Although we often think about the arithmetic operations in terms of producing particular answers, each operation also produces a signature pattern. This invariant pattern emerges when the values of the operands are allowed to vary within a particular class of number (e.g., positive integers). Figure 1 illustrates the signature patterns for the arithmetic operations for the class of positive integers. For example, addition shows a pattern in which the answer increases as either operand increases. The pattern is also completely parallel; all the curves have the same slope. Subtraction also shows a parallel pattern, but the effect of increasing the operands is quite different; increasing one operand decreases the answer, whereas increasing the other operand increases the answer. We propose that as children and adults work with an arithmetic operation, they encode its regularities and eventually come to represent particular regularities of each operation's signature pattern. Over time, these regularities, which we refer to as principles, are stored as part of the representation of the operation.

Principle definitions. For each of the four basic arithmetic operations, we propose that people represent four principles. This set of principles is structurally analogous to principles that constitute the intuitive representation (e.g., Dixon \& Moore, 1997; Dixon \& Tuccillo, 2001) and would, therefore, be useful in a structure-mapping pro- cess. Because the basic operations are initially learned with positive integers, we focused on principles for this class of number. A description of each principle for each operation can be seen in Figure 1.

The monotonicity (Mono) principle specifies whether, as the operands change in a single direction, the answers also change in a single direction. The basic arithmetic operations all have the same value for the Mono principle. That is, this principle, unlike the others considered below, is constant across all the operations children learn in grade school. Therefore, we predict that this principle will develop early in a child's representation of each operation. To illustrate the Mono principle, consider a pattern of answers that violates it: $14+15=35,14+17=37$, or 14 $+19=29$. Increasing the second operand (i.e., from 15 to 17 , from 17 to 19 ) does not always result in the same direction of change in the answers; 35 to 37 is an increase, 37 to 29 is a decrease. Therefore, the pattern of answers is not monotonic.

The relationship-to-operands (RO) principle specifies the relationship between the answer and the operands. This principle takes on different values for the different operations. For addition and multiplication, the RO principle specifies that the answer must be larger than either of the two operands. For subtraction and division, the RO principle specifies that the answer must be smaller than the minuend or the dividend, respectively. This principle can be evaluated for any of the operations by examining a single problem. That is, children are exposed to this principle for a particular operation each time they correctly solve a problem with that operation. Therefore, we expect this principle to develop fairly quickly. As an example of this principle, consider a single addition problem that violates the RO principle, $8+6=4$. Obviously, this problem does not have the correct relationship between the answer and the operands; the answer is smaller, rather than larger, than the operands.

The direction-of-effect (DE) principle specifies whether changes in one operand result in increases or decreases in the answers. This principle also takes on different values for the different operations. For addition and multiplication, increases in either operand result in increases in the size of the answer. For subtraction and division, increasing the minuend or the dividend increases the answers; increasing the subtrahend or the divisor decreases the answer. Children will only be exposed to this principle when the solutions to two or more problems are compared. The following two problems, considered together, violate the DE principle for addition: $14+15=46 ; 14+17=44$. As the second operand increases (i.e., from 15 to 17), the answer should increase rather than decrease.

Finally, the Slopes principle specifies whether the effect of increasing the size of an operand is the same regardless of the value of the other operand. As can be seen in Figure 1, addition and subtraction both specify parallel patterns of answers. Multiplication and division specify nonparallel patterns of answers (i.e., there is an inter- 


\section{Signature Patterns}

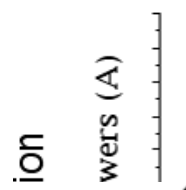

\section{Signature Patterns}
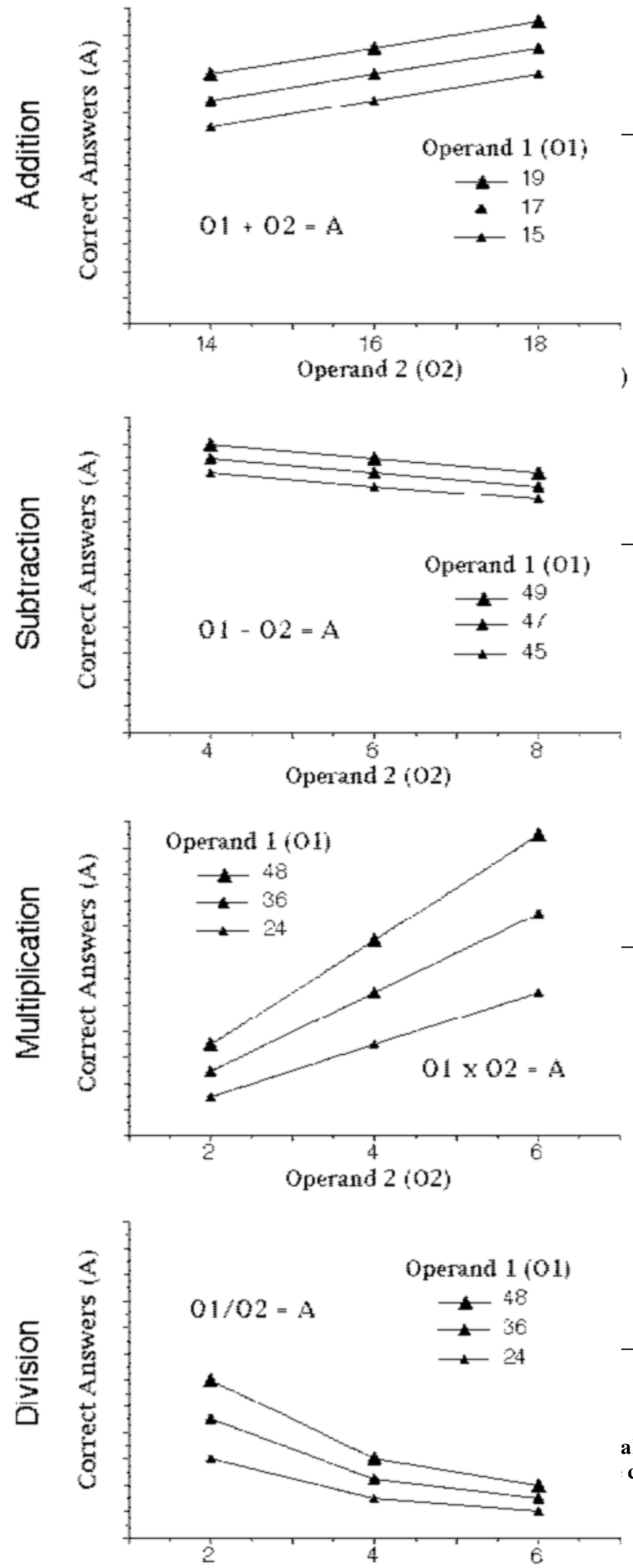

\section{Principle Descriptions}

Monotonicity: As the operands change in a single direction, the answers change in a single direction.

Relationship-to-Operands: The answer must be larger than either operand.

Direction-of-Effect: Increasing either operand increases the answer.

Slopes: The effect of one operand does not depend on the value of the other.

Monotonicity: As the operands change in a single direction, the answers change in a single direction.

Relationship-to-Operands: The answer must be smaller than the minuend (O1).

Direction-of-Effect:

a) Increasing the minuend (O1) increases the answer.

b) Increasing the subtrahend $(\mathrm{O} 2)$ decreases the answer.

Slopes: The effect of one operand does not depend on the value of the other.

Monotonicity: As the operands change in a single direction, the answers change in a single direction.

Relationship-to-Operands: The answer must be larger than either operand.

Direction-of-Effect: Increasing either operand increases the answer.

Slopes: The effect of one operand depends on the value of the other.

Monotonicity: As the operands change in a single direction, the answers change in a single direction.

Relationship-to-Operands: The answer must be smaller than the dividend (O1).

\section{Direction-of-Effect:}

a) Increasing the dividend (O1) increases the answer.

b) Increasing the divisor $(\mathrm{O} 2)$ decreases the answer.

Slopes: The effect of one operand depends on the value of the other. 
action between the operands). Children will only be exposed to this principle when they compare effects across two or more pairs of problems.

Two lines of research support the possibility that children and adults detect and represent the regularities that we call principles. First, past work has shown that people represent other regularities about the arithmetic operations. For example, Krueger showed that people represent the odd-even or parity relationships between the operands and answers for addition (Krueger \& Hallford, 1984) and multiplication (Krueger, 1986). People rejected violations of the parity relationship (i.e., $3 \times 4=13$ ) more quickly than nonviolations (i.e., $3 \times 4=14$ ). Other relationships within multiplication, such as the case of five rule (i.e., if either multiplicand is 5 , the product must end in 5 or 0; Lemaire \& Reder, 1999) and the effect of zero as a multiplicand (e.g., Miller, Perlmutter, \& Keating, 1984), also appear to be represented. Second, developmental research on children's strategy selection within an operation has shown that children have rich representations of each operation. This research focuses on the procedures children use to perform an operation such as addition $(6+3=$ ?), rather than on selecting an operation to solve a word problem. For example, research on addition strategies has shown that children do not generate new addition strategies that conflict with the goals of addition (see Rittle-Johnson \& Siegler, 1998). Given that people have representations that guide their strategy selection within an operation, it seems likely that they would represent information that would be useful in selecting an operation.

\section{Interaction With Developmental History}

Between-operations history. We expect people's developmental history with arithmetic to influence their representation of the operations. Although the details of an individual's developmentalhistory with arithmetic may be unique, the major structural aspects of that history are remarkably well defined and invariant because of clearly delineated standards in educational curricula (National Council of Teachers of Mathematics, 1989). The operations are learned in pairs; addition and subtraction are learned as a pair, as are multiplication and division. Addition and subtraction are introduced in kindergarten or first grade. Addition is learned first and is used as a basis for explaining subtraction. Instruction on addition and subtraction continues throughout early elementary school. Multiplication and division are introduced around third grade, and instruction continues through fifth grade. Multiplication is introduced first and is used to explain division.

Given that addition and multiplication are learned first in their respective pairs and are used as a basis for explaining the other member of the pair (i.e., subtraction and division), we expect addition and multiplication to have richer representations. Across pairs, we expect addition to be more richly represented than multiplication, because of its longer developmental history. Children spend 2 or
3 years working with addition before multiplication is introduced. Analogously, subtraction is predicted to have a richer representation than does division.

Within-operation history. Within each operation, the principles are predicted to develop in a particular order on the basis of (1) whether the principle must be differentiated for each operation and (2) the degree to which children experience the principle. Unlike the other principles, Mono does not need to be differentiated for each operation, because all the operations produce monotonic patterns. Therefore, we expect that for all operations, children will first develop a representation of the Mono principle. The remaining principles must be differentiated for each operation. As was mentioned in the Principle Definitions section, the principles differ in terms of how often information is available about them. Information about RO is available each time the child solves a single problem. Information about DE is available when two problems are compared. Children are exposed to information about Slopes when they compare pairs of problems. Therefore, we expect the principles to be acquired in the following developmental order within each operation: Mono, RO, DE, and finally Slopes.

Note that the present research focuses on formal arithmetic operations on numbers, rather than on physical operations on sets of discrete objects. There is considerable evidence that very young children understand many of the consequences of transforming sets of physical objects but that understanding of formal mathematics develops only after considerable schooling (see Geary, 1995).

\section{EXPERIMENT 1}

To evaluate whether people represent the principles, we asked participants to rate sets of completed problems for each operation. The answers to each set of problems were supposedly generated by a student who was just learning that arithmetic operation. The participants were asked to rate how good or bad each student's attempt at that operation was. Figure 2 shows examples of four different trials for addition. For each trial, the participants were told that a different hypothetical student had solved all nine of the problems in the panel. The participants were asked to rate how good or bad the student's overall attempt at addition was (i.e., the participants made a single rating for each panel in Figure 2).

The problem sets were independently manipulated in two ways. First, we manipulated whether or not the problem set violated a principle. Second, we manipulated the amount by which each problem set was wrong (i.e., the average absolute difference between the presented answers and the correct answers). For example, the upper panels of Figure 2 show examples of addition in which no principles are violated, although the answers are incorrect. The lower panels of Figure 2 show two examples of problem sets that violate the DE principle (i.e., as the size of the operands increases, the size of the answers decreases). Notice that the problem sets that violate DE re- 

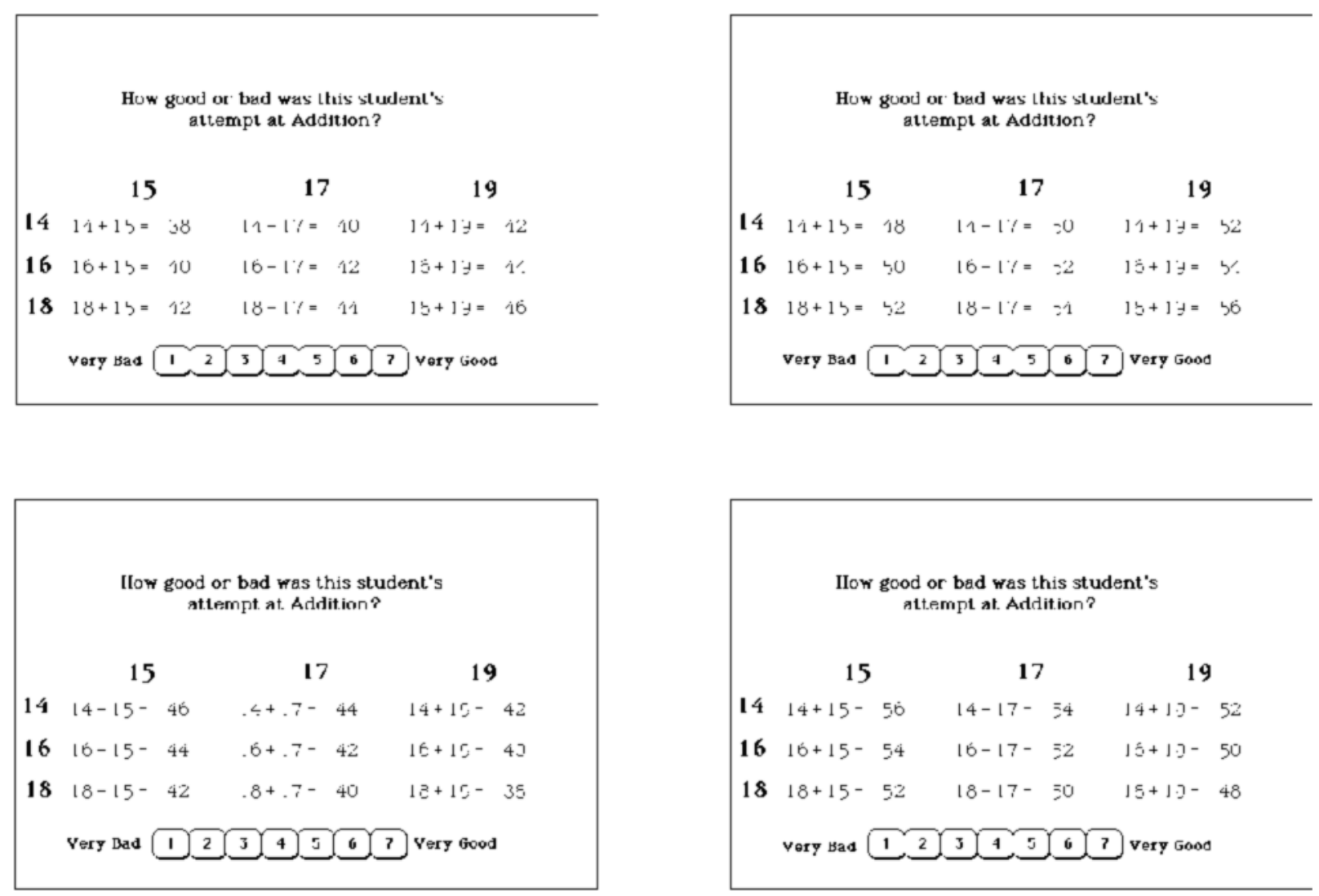

Figure 2. Each panel shows a different trial for addition. The rating scale is shown at the bottom of each display. For each panel, the participants were asked to rate the hypothetical student's attempt at math. The upper two panels show problems that are incorrect but do not violate any principles. The lower two panels show problems that violate the direction-of-effect principle. Panels on the left side are equally wrong; presented answers are an average absolute distance of 9 units from the correct answers. Panels on the right side are also equally wrong, 19 units from the correct answers.

spect all the other principles (i.e., the answers are larger than the operands, the patterns are monotonic and have equal slopes).

The amount-wrong manipulation is also demonstrated in Figure 2. The presented answers in the two problem sets on the left side of the figure are the same average distance, 9 units, from the correct answers. The presented answers in the two problem sets on the right side of the figure are further from the correct answers: Both problem sets have an average distance of 19 units. Amount wrong and principle violation were manipulated in a factorial design for each of four principles for each operation.

This set of manipulations allows for a very straightforward prediction. If the participants represent a principle, problem sets that violate that principle should be rated as worse attempts at the operation than are equally incorrect problems. We also expect that, consistent with past research showing strong effects of split, problem sets with presented answers that are further from the correct answers will be rated as poorer attempts at the operation.

In the first experiment, college students were asked to rate problems sets for each arithmetic operation. We pre- dicted they would rate problem sets that violated principles as poorer attempts at the operation. On the basis of their developmental history with the operations, we also predicted that the principle representation of (1) addition would be more complete than that of subtraction or multiplication, and that the principle representation of (2) subtraction and multiplication would be more complete than that of division. We predicted that the principles should be acquired in the order Mono, RO, DE, and Slopes, and that the operations would differ in terms of how many of the principles had accumulated.

\section{Method}

\section{Participants}

Seventeen male and 12 female college students (mean age in years $=18.79, S D=0.82$ ) participated as one option for obtaining extra credit for a psychology course.

\section{Materials}

Problem sets were presented on a Macintosh computer using HyperCard. The keyboard was altered so that seven keys were labeled with the numerals 1 through 7 . These keys were chosen so as to be equidistant from the center of the space bar. The participants were 
required to press the space bar to advance to the next trial. Only the center of the space bar could be used; the ends were shielded by tag board. The computer recorded both the participant's rating and the response time.

\section{Procedure}

The participants were tested individually in two sessions lasting between 20 and $35 \mathrm{~min}$ each. They were told that they would see a number of different sets of problems and that each set of problems had been solved by a different hypothetical student who was just learning that arithmetic operation. The participants were asked to rate, on a scale of 1 to 7 , each student's attempt at the arithmetic operation. One end of the scale (1) was marked as very bad, and the other (7) as very good (see Figure 2). The participants were informed that they were being timed. They were asked to take as long as they needed to make a carefully considered rating but not to take longer than necessary. The participants pressed the space bar to advance to the next problem. Four practice trials were completed before starting the experimental trials. At the end of the experiment, the participants were debriefed.

\section{Design}

The same nine problems were used for all the trials within a single arithmetic operation. The problems were generated by factorially combining two sets of three operands. One set of operands formed the rows, and the other formed the columns. The operand for each row and column was displayed in bold along the margin (see Figure 2). The participants were shown that each row of problems was formed by combining the operand for the row with the operand for each column.

For each arithmetic operation, four main subdesigns were constructed, one for each of the four principles. ${ }^{1}$ For each subdesign, we manipulated (1) whether or not the principle was violated and (2) the amount by which the answers were wrong. The amount wrong was calculated as the average absolute difference between the presented and the correct answers. The levels of amount wrong were selected to simultaneously fulfill two objectives. First, we wanted to manipulate amount wrong as strongly as possible. Second, we wanted the amount wrong to be a similar proportion of the answers for each operation. The different operations produce answers of different magnitudes. For example, multiplication produces much larger answers than addition. This is potentially important because the same deviation from the correct answer is not subjectively the same when the correct answers differ greatly in magnitude (Stevens, 1974). For example, being off by 5 seems quite serious if the correct answer is 12 , but considerably less so if the correct answer is $272 .^{2}$

Problem sets were constructed so that only the principle of interest was violated by the problem set; the other principles were respected. Examples of problem sets that violate each principle are shown for each operation in the Appendix. ${ }^{3}$ The principles were violated analogously for each operation. The trials for each of the four different arithmetic operations were presented in blocks (i.e., all the addition problems were presented in a single block). The order of the blocks and the order of the trials within each block were determined randomly. There were 38 trials for each operation.

For each arithmetic operation, a single set of nine problems was used. Whether or not the parity of the presented answers was the same as the correct answers was held constant within each subdesign. Problem sets had the same parity as the correct answers, the opposite parity, or a mixture of the two. To control for differential weighting of correct answers, all the presented answers were incorrect.

Three of the four practice trials differed only in their amount wrong and covered the range of amount wrong for the experimental trials for that operation. Problem sets presented on these trials did not violate any of the principles. The fourth problem set showed a pattern in which all the answers were the same number. This vio- lates perhaps the most basic principle of arithmetic operations; changes in operands will yield different answers. Practice problems were presented in random order.

\section{Results}

The participants made their ratings fairly quickly. Mean response time in seconds was $6.30(S D=2.84)$, $7.02(S D=3.87), 7.84(S D=5.04)$, and $9.61(S D=4.65)$ for addition, subtraction, multiplication, and division, respectively. Response times are reported in order to give a sense of how quickly the participants did the task; the manipulations were not predicted to produce differences in response times. The analyses focused on the participants' ratings. For addition and multiplication, we conducted a 4 (principle) $\times 2$ (principle violation) $\times 2$ (amount wrong) analysis of variance (ANOVA) on ratings of goodness. For subtraction and division, we conducted a 5 (principle) $\times 2$ (principle violation) $\times 2$ (amount wrong) ANOVA. (The additional principle level for subtraction and division comes from the DE principle, which takes on two values for these operations, one value for each operand.) The results from the analogous multivariate ANOVA (MANOVA) model are reported when the sphericity assumption is violated. Within each operation, planned comparisons were conducted to test whether the participants detected violations of each principle. ${ }^{4}$

\section{Ratings of Attempts at Each Operation}

Addition. The left panel in the top row of Figure 3 shows the mean ratings for addition problem sets that violated (gray bars) and did not violate (black bars) each of the four principles. Problem sets that violated a principle were rated as poorer attempts at addition $[F(1,28)=$ 36.58]. This effect did not differ across principles $(F<$ 1). Planned comparisons confirmed that the participants detected violations of all the principles for addition $[F(2,56)=11.10, F(1,28)=12.00, F(1,28)=11.89$, and $F(1,28)=7.06$, for Mono, RO, DE, and Slopes, respectively].

Subtraction. The left panel in the second row of Figure 3 shows the mean ratings for subtraction. The effect of violating a principle was significant $[F(1,28)=12.71]$, but depended on which principle was violated $[F(4,112)=$ 4.54]. Problem sets that violated Mono, RO, DE for the subtrahend, and Slopes were rated as poorer attempts at subtraction $[F(2,56)=6.79, F(1,28)=20.38, F(1,28)=$ 20.35 , and $F(1,28)=5.61$, respectively]. However, the participants did not detect violations of DE for the minuend $(F<1)$.

Multiplication. The left panel of the third row of Figure 3 shows the participants' ratings of multiplication problem sets. The participants rated problem sets that violated a principle as poorer attempts at multiplication $[F(1,28)=41.38]$, but the effect depended on the principle [MANOVA, $F(3,26)=3.98$ ]. Planned comparisons showed that violations of Mono, $\mathrm{RO}$, and $\mathrm{DE}$ were detected $[F(2,56)=14.83, F(1,28)=27.33$, and $F(1,28)=28.89$, respectively] but violations of Slopes were not $(F<1)$. 


\section{Principle Violations}
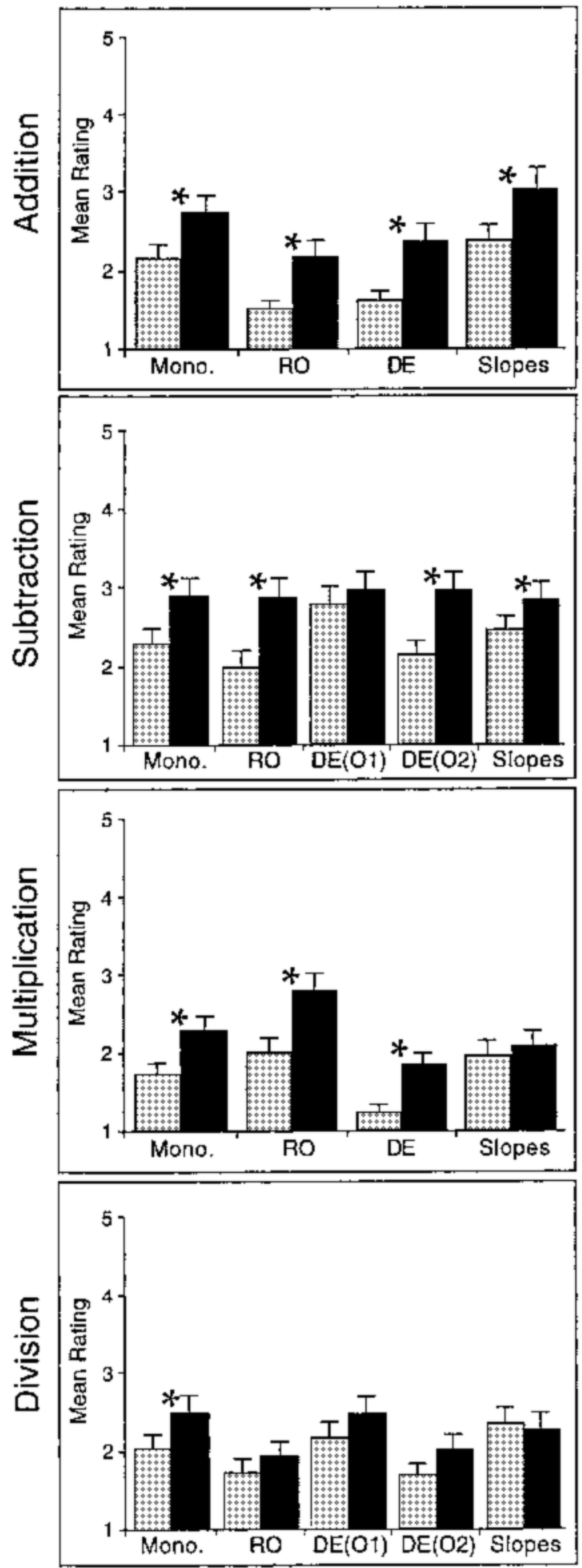

Amount Wrong
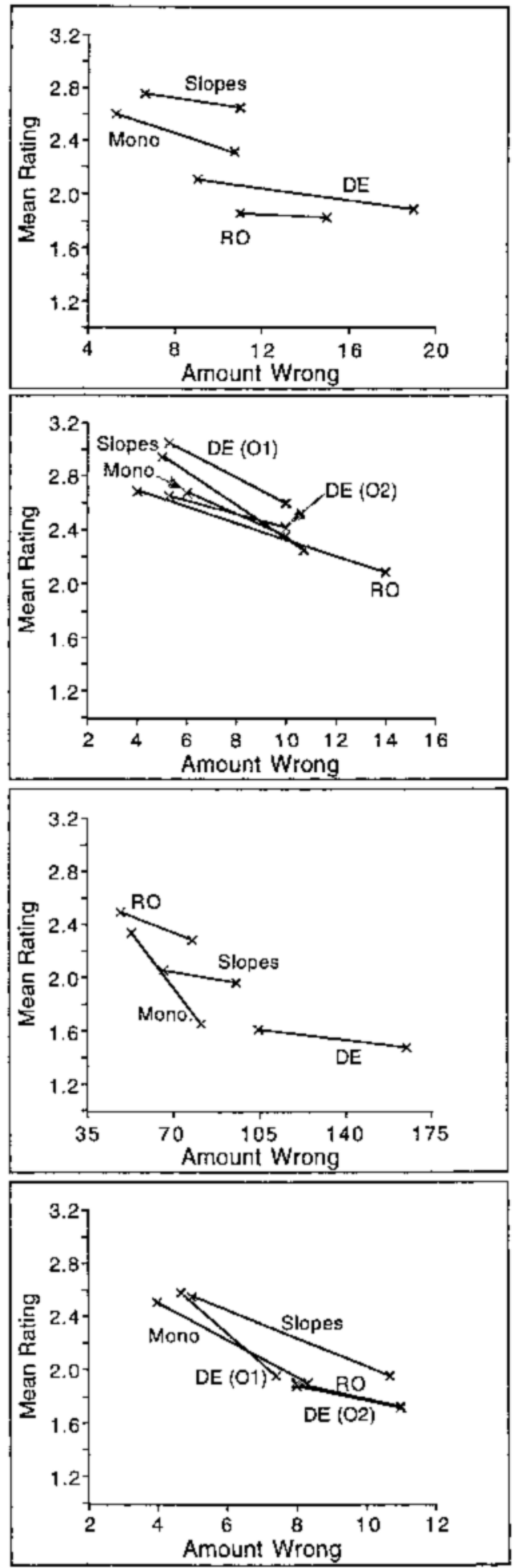

Figure 3. Panels in the left column show mean judgments for problem sets that violate a principle (gray bars) and problem sets that are equally wrong but do not violate a principle (black bars). The principles are shown along the horizontal axis. Asterisks indicate a significant effect of violating the principle. Standard error bars are also presented. The right columns show mean judgments for each principle plotted as a function of amount wrong. The top row shows the results for the addition subdesign. The results for subtraction, multiplication, and division are shown in the second, third, and fourth rows, respectively. 
Division. The participants' mean ratings of attempts at division are in the bottom left panel of Figure 3. Problem sets that violated a principle were rated as poorer attempts at division $[F(1,28)=6.67]$, but this effect depended on the principle [MANOVA, $F(4,25)=3.31$ ] Planned comparisons showed that only problem sets that violated Mono were rated as poorer attempts at division $[F(2,56)=6.26]$. Violations of the other principles were not reliably detected $[F(1,28)=1.90, F(1,28)=2.76$, $F(1,28)=3.30$, and $F<1$, for RO, DE for the dividend, $\mathrm{DE}$ for the divisor, and Slopes, respectively].

Effect of amount wrong. The right panels in Figure 3 show mean ratings for each operation as a function of amount wrong (averaged over principle violation). For each operation, the participants rated problem sets that were more distant from the correct answers as poorer attempts [addition, $F(1,28)=4.50$; subtraction, $F(1,28)=$ 15.67; multiplication, $F(1,28)=5.18$; and division, $F(1,28)=18.67]$.

\section{Discussion}

Consistent with the hypothesis that people represent the principles, the participants were sensitive to principle violations. Problem sets that violated a principle were rated as poorer attempts at the operation. We also observed the predicted effects of children's developmental history with each operation. The representation of addition appeared to be the most complete, containing all the principles. The representation of subtraction was only slightly less complete; all the principles except the DE principle for the minuend appeared to be represented. The participants did not detect violations of the relationship between changes in the minuend and changes in the answer. Similarly, the representation of multiplication appeared to be less complete than that of addition; the participants did not represent the Slopes principle for multiplication. Division was more poorly represented than either subtraction or multiplication; only violations of the Mono principle were rated as poorer attempts at division.

The pattern of principles across operations is consistent with the predicted developmental ordering of the principles. The representation of division contains only one principle, Mono, which is predicted to develop first. Multiplication is missing only one principle, Slopes, which can be expected to develop last. The representation of subtraction is missing a single principle, DE for the minuend. DE is predicted to develop late in the sequence and is the only principle that takes on two values for a single operation.

Regardless of whether the principle representation of an operation was extensive (e.g., addition) or meager (e.g., division), the participants rated problem sets that were further from the correct answers as poorer examples of that operation. The participants clearly had a sense of how much the presented answers deviated from the correct answers for all the operations. This shows that differences in detecting violations of the principles among the operations are not due to differences in participants' ability to approximate the correct answers for different operations.

\section{EXPERIMENT 2}

The results of the first experiment are consistent with the hypothesis that college students represent principles of the four arithmetic operations and that the quality of these representations reflects predictable aspects of their developmental history with mathematics. In the second experiment, we sought to extend this evidence to younger students, eighth graders, and replicate the effects observed in the first experiment with a second sample of college students.

Like college students, eighth graders have considerable experience with the arithmetic operations. However, eighth graders have 4 or 5 fewer years of experience with the operations and are not as mathematically sophisticated as college students in terms of their problem-solving skills. Therefore, although eighth grade students are predicted to have principle representations for the arithmetic operations, their representations will not be as extensive as those of college students.

\section{Method}

\section{Participants}

Forty-one eighth grade students, 19 male and 23 female, from local parochial and public schools (mean age $=14.44$ years, $S D=$ 0.67 ) and 25 college students, 15 male and 10 female (mean age = 19.76 years, $S D=1.36$ ), participated.

\section{Design and Procedure}

The design of the second experiment was analogous to that of the first, with the following exceptions. First, because the ratings in the first experiment tended to be toward the bottom of the scale (i.e., the participants rated students' performance very harshly), we changed the description of the upper anchor of the scale from very good to pretty good. The participants in the second experiment were also explicitly told that all the answers were incorrect but that some of the attempts were better than others. Both these changes were expected to increase the range of responses on the scale. Finally, because of time constraints, eighth graders completed either the addition and subtraction blocks $(n=21)$ or the multiplication and division blocks $(n=20)$ in a $30-40$ min session. College students completed the full design in two 20-35 min sessions.

\section{Results}

The analyses focused on (1) whether participants in each grade detected principle violations and (2) the effect of amount wrong (i.e., split). We conducted a 2 (grade) $\times 4$ (principle) $\times 2$ (principle violation) $\times 2$ (amount wrong) ANOVA for addition and multiplication and a 2 $($ grade $) \times 5$ (principle $) \times 2$ (principle violation $) \times 2$ (amount wrong) ANOVA for subtraction and division.

\section{Addition}

The top panels of Figure 4 show the mean ratings for attempts at addition; ratings for eighth graders are on the left side, ratings for college students are on the right. Eighth graders and college students both rated problem sets that 
8th Grade
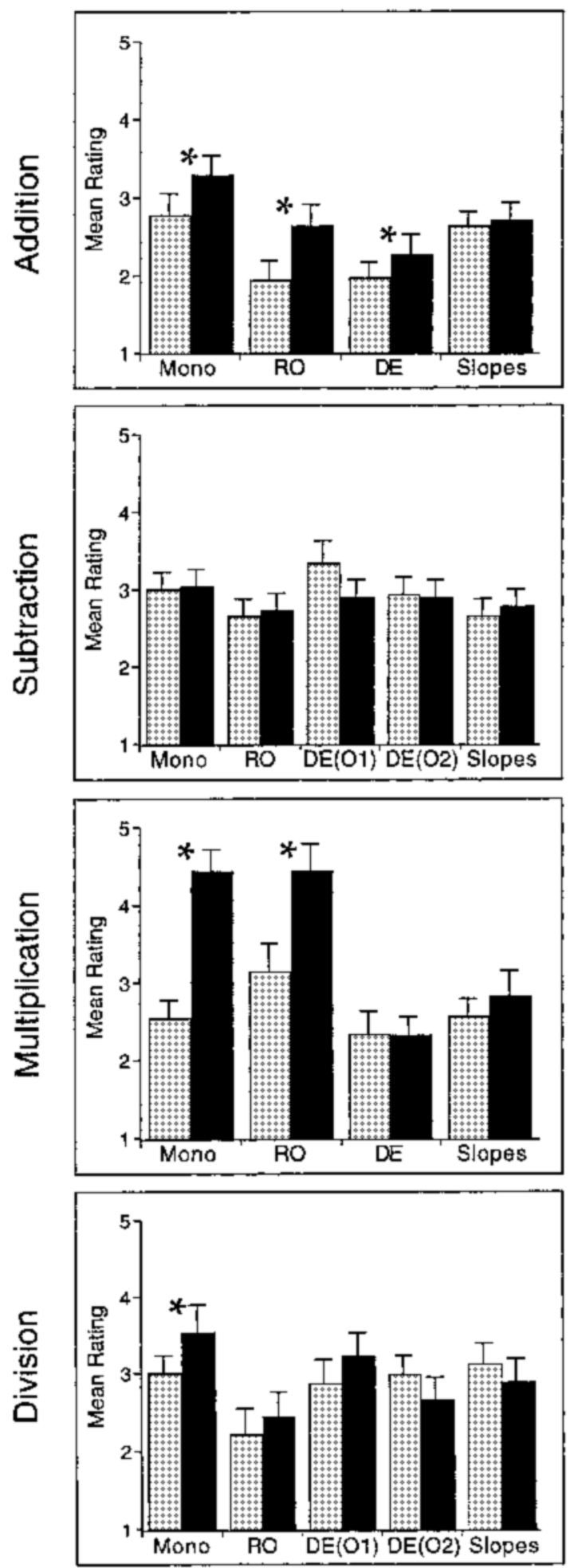

College
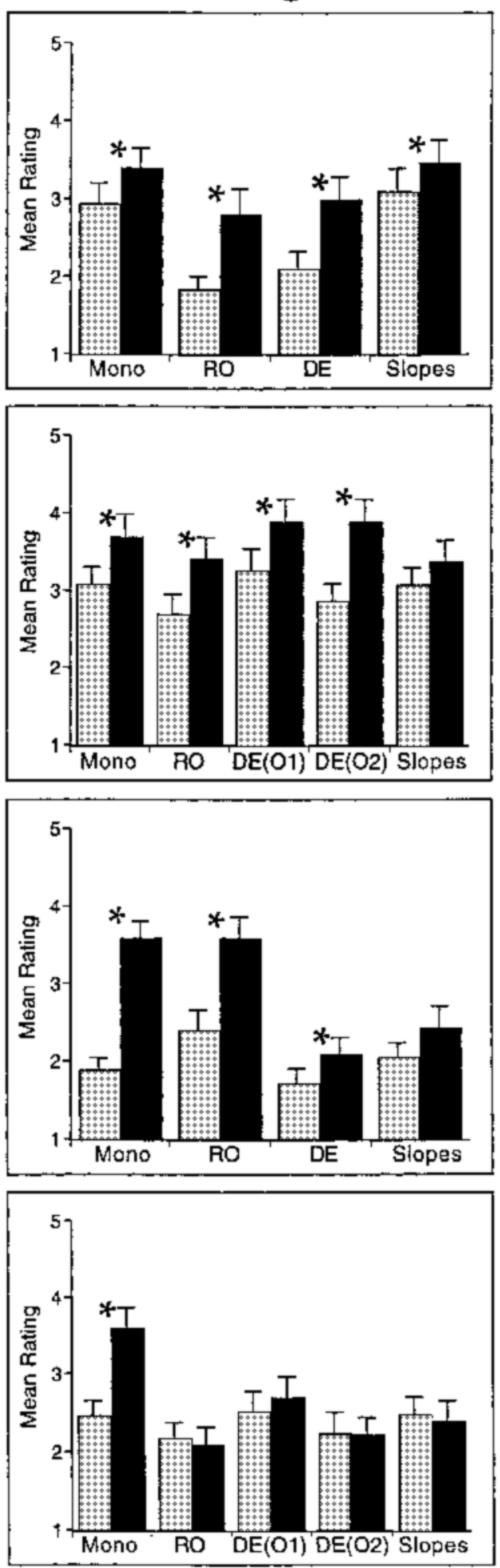

Figure 4. Panels in the left column show mean judgments of the eighth grade students for problem sets that violate a principle (gray bars) and problem sets that are equally wrong but do not violate a principle (black bars). The right columns show the mean judgments for the college students. The principles are shown along the horizontal axis. Asterisks indicate a significant effect of violating the principle. Standard error bars are also presented. The top row shows results for the addition subdesign. Results for subtraction, multiplication, and division are shown in the second, third, and fourth rows, respectively. 
violated a principle as poorer attempts at the operation $[F(1,44)=45.31]$. Planned comparisons showed that the only difference between the age groups for addition was that eighth graders did not detect violations of Slopes $(F<1)$ but the college students did $[F(1,24)=5.05]$.

\section{Subtraction}

The second row in Figure 4 shows the mean ratings for attempts at subtraction. The effect of violating a principle was very different for the eighth graders and the college students $[F(1,44)=12.33]$. The eighth graders did not reliably detect principle violations $(F<1)$, and this did not depend on which principle was considered [MANOVA, $F(1,17)=1.11]$. The college students did not reliably detect violations of Slopes $[F(1,24)=3.60$, n.s. $]$ but did detect violations of all the other principles [Mono, $F(2,48)=$ 5.52 ; RO, $F(1,24)=6.47$; DE for the minuend, $F(1,24)=$ 5.37; DE for the subtrahend, $F(1,24)=33.31]$.

\section{Multiplication}

The third row of panels in Figure 4 shows mean ratings for multiplication. The effect of violating a principle depended on which principle was violated $[F(3,129)=$ $10.46]$. Both the eighth graders and the college students detected violations of Mono $[F(2,38)=6.10, F(2,48)=$ $8.88]$ and $\mathrm{RO}[F(1,19)=14.70, F(1,24)=39.27$, respectively]. The eighth graders did not detect violations of either DE or Slopes $\left(F_{\mathrm{S}}<1\right)$. The college students detected violations of DE $[F(1,24)=8.40]$, but not of Slopes $[F(1,24)=3.83]$.

\section{Division}

The bottom row of panels in Figure 4 shows the mean ratings for attempts at division. The effect of violating a principle depended on which principle was violated $[F(4,172)=4.36]$; this interaction did not depend on grade $(F<1)$. Planned comparisons revealed that for both grades only violations of the Mono principle were rated as poorer attempts at division $[F(2,38)=4.37$, $F(2,48)=6.18$, for the eighth grade and the college students, respectively].

\section{Effect of Amount Wrong}

The effect of amount wrong was significant for all the operations $[F(1,44)=32.47, F(1,44)=61.08, F(1,43)=$ 32.86 , and $F(1,43)=32.47$, for addition, subtraction, multiplication, and division, respectively]. The effect of amount wrong depended on grade for subtraction $[F(1,44)=5.27]$, but not for the other operations (all $\left.F_{\mathrm{s}}<1\right)$. However, both grades showed significant effects of amount wrong for subtraction [eighth grade, $F(1,20)=$ 58.92; college, $F(1,24)=14.29$ ]

\section{Discussion}

The results of the second experiment replicated and extended the findings of the first experiment. Both age groups were sensitive to principle violations, but the col- lege students appeared to have a more extensive principle representation than did the eighth graders. The differences between the two age groups are consistent with the predicted effects of children's developmental history with the operations and principles. The college students' representations did not just contain more principles; they contained principles predicted to develop later in the sequence. For example, the college students had a more extensive representation of addition than did the eighth grade students. The difference between their two representations was that the college students represented the Slopes principle, the last principle in the sequence, whereas the eighth graders did not. Likewise, for multiplication, the eighth grade students had only acquired the first two principles in the sequence, Mono and RO. The college students had acquired the next principle in the sequence, DE, but had not yet acquired the final principle, Slopes.

The differences between operations within each grade are also consistent with the predicted effects of developmental history. For both grades, the representation of addition was more extensive than that of either subtraction or multiplication. Similarly, the representation of multiplication was more complete than that of division. For the college students, but not for the eighth grade students, the representation of subtraction was more extensive than that of division. Why the eighth grade students did not represent any of the principles for subtraction is not clear. We address possible explanations and connections to other findings in the General Discussion section. Note that the differences in the representations across operations also follow the predicted order of the acquisition of the principles. Operations that have better representations contain later developing principles, not just more principles.

We propose that children and adults represent the principles for the different operations and that the principles are acquired through experience in using the different operations. The developmental ordering of the principles within and between operations and across grades is consistent with this hypothesis. An alternative explanation is that the participants induced these relationships during the task. That is, by repeatedly looking at problem sets during the experimental session, participants might identify regular relationships and then make lower ratings when these relationships are violated. This explanation makes a very clear prediction: Because the operations are equally regular with regard to the principles, there should be no between-operations differences, such as those predicted by developmental history. For example, subtraction and division are equally regular with regard to the RO principle, and therefore, the induction hypothesis predicts that participants should be equally likely to induce the relationship for both operations. However, the college students were sensitive to violations of this relationship for subtraction, but not for division. All the between-operations differences cause difficulty for the induction hypothesis.

One might suggest that the induction hypothesis could be saved by proposing that differences in other aspects of 
the pattern make induction differentially difficult. For example, the vast majority of the problem sets presented for subtraction had a parallel pattern; those for division had a nonparallel pattern (so as to be consistent with the normative pattern for each operation). This difference might affect people's ability to induce other relationships within the pattern (e.g., the RO principle). However, this explanation makes further predictions that are not confirmed by the data. Specifically, addition and multiplication have the same parallel/nonparallel difference as subtraction and division, and therefore, inducing RO should be difficult for multiplication. But the college students were sensitive to violations of the RO principle (and the more complex DE principle) for both addition and multiplication. Therefore, the parallel/nonparallel difference cannot explain the observed differences between subtraction and division. Despite the fact that the induction hypothesis has difficulty explaining aspects of the data pattern, ${ }^{5}$ we conducted a third experiment to test additional aspects of the principle representation hypothesis, using a methodology in which the central prediction was inconsistent with induction.

\section{EXPERIMENT 3}

The first two experiments focused on developmental differences among the operations in terms of the extensiveness of the principle representations. Operations with longer developmental histories had more extensive principle representations. The third experiment focused on differences in the quality of these principle representations. Not only should an operation with a longer developmental history have more principles in its representation, the principles that are present should be represented more strongly. For example, college students appear to represent the RO principle for both addition and multiplication, but because addition has a longer developmental history than multiplication, the representation of this principle should be stronger for addition.

In the third experiment, we asked the participants to imagine that they were playing a game with another person. The participants were told that the other player was thinking of one of the four arithmetic operations and that the other player would give them a hint to help them guess which one it was. It was specified that the arithmetic operation involved two operands. The participant read a single statement about the operation, which was attributed to the other player, and then rated how likely it was that the other player was thinking about each of the operations: addition, subtraction, multiplication, and division. For example, the statement made by the imaginary player might be, "The answer is always larger than the first number in the problem." The participant would then rate the likelihood that this statement was intended to describe each operation.

The participants rated the likelihood that the other player was describing each of the four operations for eight separate statements. The statements are presented in Figure 5. Four of the statements described whether the answer was greater or less than one of the operands and were therefore relevant to the RO principle. Four of the statements described whether changing an operand increased or decreased the answer and were therefore relevant to the DE principle.

We expected two factors to contribute to the participants' likelihood ratings. First, the participants would evaluate whether a statement was "true" for an operation. That is, the participants might construct an example addition problem and check whether or not the statement held for that problem. Second, we expected that if the participants represented a principle for an operation, statements that were semantically consistent with that principle would activate it and make the statement seem more appropriate for that operation. For example, when a participant encodes the meaning of the statement, "The answer is always larger than the first number in the problem," it will activate the RO principle for addition, thereby increasing the participant's impression that the statement describes addition. Participants' ratings of the degree to which a statement implies an operation will be a weighted average of (1) the perceived "truth" of the statement for the operation and (2) the strength of the relevant principle for the operation.

Given this analysis, the principle representation hypothesis makes the following set of predictions. First, when a statement is consistent with both addition and multiplication, participants should rate the statement as more indicative of addition, because it has a longer developmental history and, therefore, better representation of the principle. For example, addition and multiplication are both consistent with (i.e., have the same truth value for) the statement, "The answer is always larger than the first number in the problem." However, addition has a longer developmental history and so will have a stronger representation of the relevant principle, RO. Therefore, this statement should seem more indicative of addition than of multiplication. Similarly, statements that directly conflict with a principle should be rated as less indicative of addition than multiplication. Analogous effects will be seen for the DE principle.

However, when a statement is consistent with both subtraction and division, effects of developmental history on the principle representation will not be observed. Recall that the first two experiments showed that college students do not represent the RO or DE principles for division. Participants' ratings of these statements for division will be solely a function of the perceived truth of the statement, because the principle information is absent. The weighted averaging model predicts that the net effect of the truth of the statement will increase because the weight of the absent principle information goes to zero in the denominator (e.g., Levin, Johnson, \& Faraone, 1984), offsetting any effects of developmental history. Therefore, participants ratings of statements relevant to $\mathrm{RO}$ and $\mathrm{DE}$ for sub- 


\section{Relationship-to-Operands}

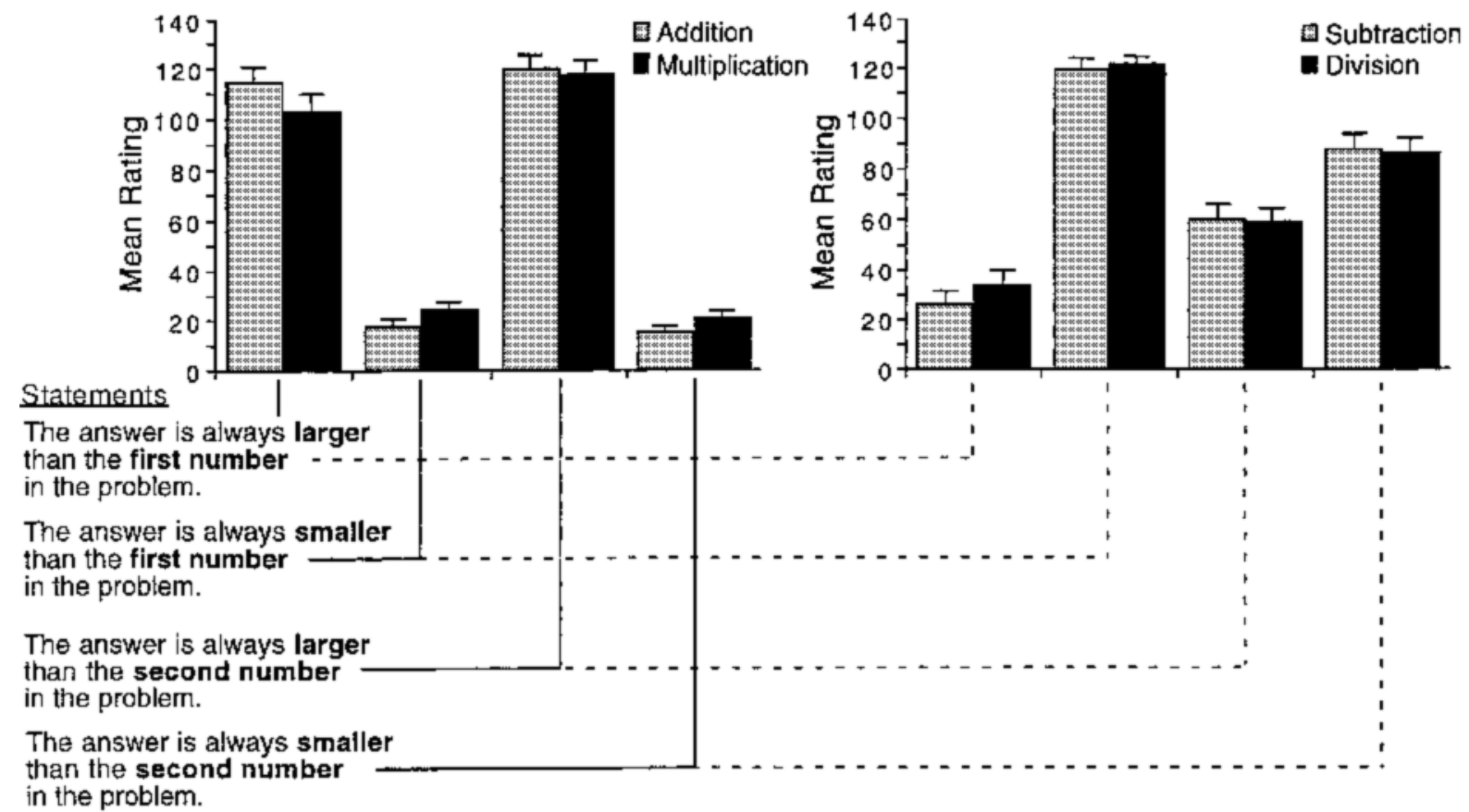

in the problem.

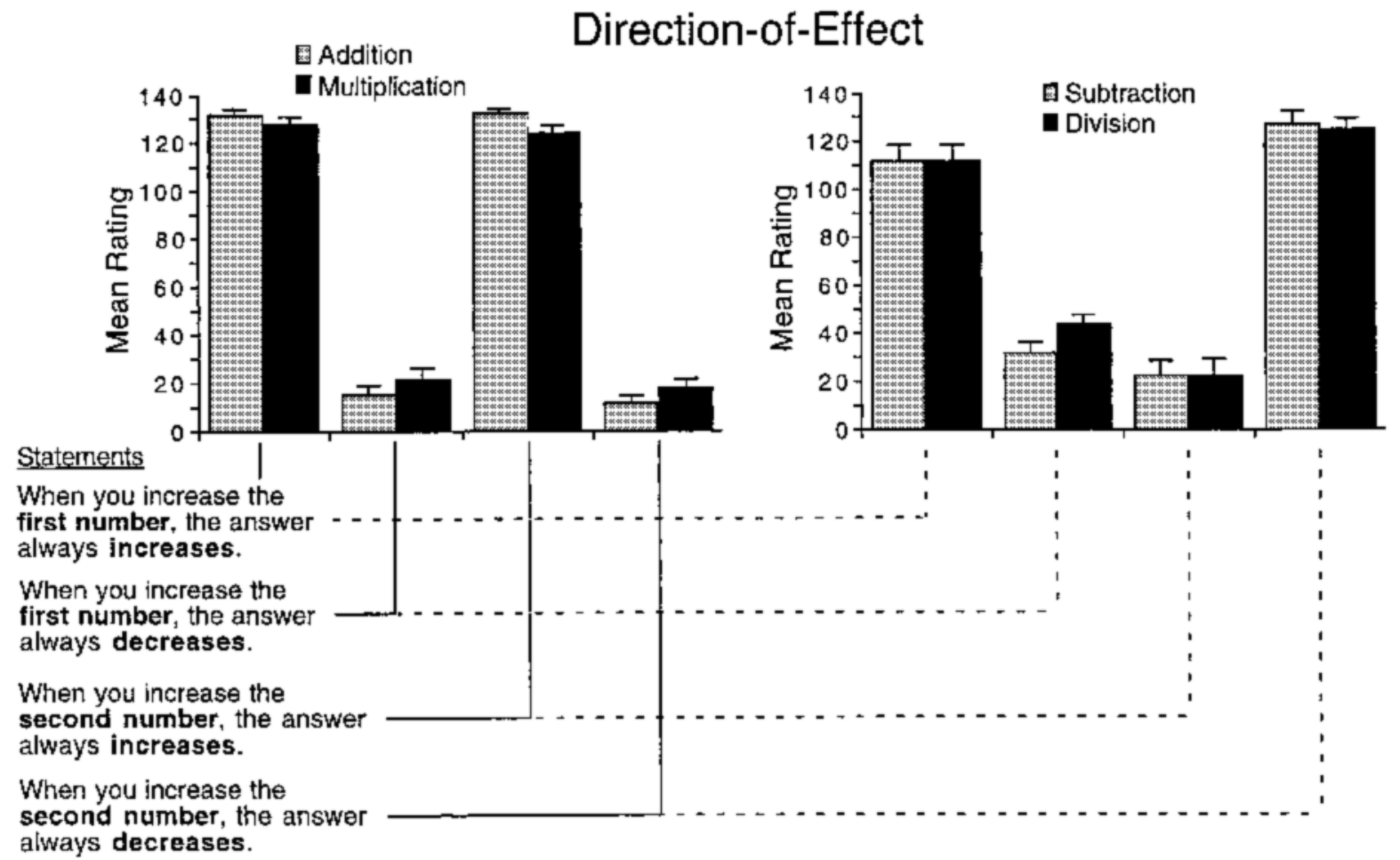

Figure 5. The top two panels show mean ratings for statements that are relevant to the relationship-to-operands principle. The four statements are presented below the panels. Ratings for addition and multiplication are on the left. Ratings for subtraction and division are on the right. Higher scores indicate that the statement was rated as more indicative of the operation. Mean ratings for statements relevant to the direction-of-effect principle are shown in the lower two panels. Standard error bars are shown for each mean rating. 
traction and division will not show effects of developmental history.

\section{Method}

\section{Participants}

Thirty-nine college students ( 27 females and 12 males) participated as one option for fulfilling a course requirement.

\begin{abstract}
Materials and Procedure
The participants were asked to imagine that they were playing a game with another person. They were told that the other player was supposedly thinking about a particular arithmetic operation and that the other player would try to communicate which one it was by making a statement about the operation. The participant was asked to read the statement made by the other player. The statement described something about how the operation functioned with twooperand problems. Pilot testing showed that participants assumed that the operands were positive integers. ${ }^{6}$ After reading the statement made by the other player, the participants were asked to rate the likelihood that the other player was describing addition, subtraction, multiplication, and division. The participants made their ratings by drawing a vertical line through the appropriate point on a 145-mm horizontal scale. One end of the scale was marked very likely; the other end was marked very unlikely. The participants were free to make their ratings for each operation in any order they wished. The participants made ratings for each of eight statements in one of five random orders. Each statement appeared at the top of a separate page of paper; the scales for each operation appeared below.
\end{abstract}

\section{Relationship to Operands}

\section{Results}

The participants' ratings of the RO statements for addition and multiplication were entered in a 2 (principle value: larger vs. smaller) $\times 2$ (operand) $\times 2$ (operation: addition vs. multiplication) ANOVA. As can be seen in the top left panel of Figure 5, the participants rated the statements that specified that the answer would be larger than an operand as indicative of addition and multiplication. Statements that specified that the answer would be smaller than an operand were rated as not indicative of addition and multiplication. The ANOVA showed a significant main effect for principle value $[F(1,38)=367.19]$, but not for operation or operand $\left(F_{\mathrm{S}}<1\right)$. However, as was predicted, the participants rated statements that were consistent with both addition and multiplication as more indicative of addition than of multiplication and statements that were inconsistent with these operations as less indicative of addition $[F(1,38)=5.24]$.

Mean ratings of RO statements for subtraction and division are in the upper right panel of Figure 5. Ratings were analyzed in a 2 (principle value: larger vs. smaller) $\times 2$ (operand) $\times 2$ (operation: subtraction vs. division) ANOVA. The effect of principle value (i.e., whether the statement specified that the answer must be larger or smaller than the operand) was significant $[F(1,38)=$ $119.85]$, but this effect was greater for the first operand than for the second $[F(1,38)=40.68]$. This interaction makes sense, because there is no logical relation between the second operand and the answer. Therefore, the effect of the value of the principle (i.e., larger vs. smaller) with regard to the second operand should be close to zero. As was predicted, in contrast to addition and multiplication, the effect of the principle value (i.e., larger vs. smaller) did not depend on the operation $(F<1)$.

\section{Direction of Effect}

A similar pattern of results is seen for the DE principle in the lower two panels of Figure 5. A 2 (principle value: positive vs. negative) $\times 2$ (operand) $\times 2$ (operation: addition vs. multiplication) ANOVA on the participants' ratings for addition and multiplication showed a significant main effect of principle value $[F(1,38)=$ 734.56]. Consistent with the principle representation hypothesis, the participants rated statements that were consistent with both addition and multiplication as more indicative of addition than of multiplication and statements that were inconsistent with both operations as less indicative of addition $[F(1,38)=8.42]$.

The lower right panel of Figure 5 shows the mean ratings of DE statements for subtraction and division. A 2 (principle value: increases vs. decreases) $\times 2$ (operand) $\times 2$ (operation: addition vs. multiplication) ANOVA showed a main effect for principle value $[F(1,38)=165.50]$. Again, as was predicted, the effect of principle value did not depend on the operation $[F(1,38)=2.18$, n.s. $]$.

\section{GENERAL DISCUSSION}

The three experiments reported here provide initial evidence for the hypothesis that children and adults represent principles that specify how the operations function. Experiments 1 and 2 showed that problem sets that violated the principles were rated as poorer attempts at arithmetic, as compared with problem sets that did not violate the principles but were the same distance from the correct answers. Both the college and the eighth grade students were sensitive to principle violations. As was predicted, operations with longer developmental histories had more extensive principle representations. Principles that must be differentiated for each operation and that are less frequently in evidence appear to develop later, both across age groups and across operations. Importantly, differences in the principle representations between age groups and across operations were not related to the ability to approximate the answers; both ages showed reliable effects of amount wrong for all the operations.

Experiment 3 showed that the quality of the principle representation depended on the developmental history of the operation. Despite the fact that the RO and DE principles take the same values for addition and multiplication, statements that are consistent with these principles for addition and multiplication were rated as more indicative of addition than of multiplication (and vice versa for conflicting statements). This pattern of results is consistent with the hypothesis that the representation of these principles is stronger for addition than for multiplication. 
Given that the first two experiments showed that the RO and DE principles were not represented for division, parallel effects were not predicted for subtraction and division. Because RO and DE were not represented for division, principle activation was predicted to be zero, and therefore, the net effect of evaluating the truth value of the statement should increase. As was predicted, the students made sensible ratings about subtraction and division, reflecting their ability to evaluate the truth value of the statements for each operation, but they did not show any reliable between-operations effects.

Both the college and the eighth grade students appeared to have a poor representation of division, and the eighth graders appeared to have an especially poor representation of subtraction. To the extent that we consider subtraction and division to be mirror images of addition and multiplication, these results seem counterintuitive. However, our findings are consistent with recent evidence that multiplication and division are represented separately (e.g., Campbell, 1999) and with evidence on the relative difficulty of problem solving when using the different operations. For example, there is strong empirical evidence that shows that solving subtraction word problems is more difficult than solving addition problems, regardless of problem type (Nesher, 1982). Likewise, problem solving with division is more difficult than with multiplication, even for students in the eighth grade and above (Reese, Miller, Mazzeo, \& Dossey, 1997). The present study suggests that one reason people may have more difficulty employing these operations is that they have poor representations of how these operations function.

A number of factors may jointly suppress the development of the principles for subtraction and division. First, subtraction and division are introduced after addition and multiplication, respectively, and are explained as a way to "undo" those operations, rather than as important and unique operations themselves. Second, the principles for subtraction and division contain inverse relationships (e.g., the larger the divisor, the smaller the answer), which tend to be more difficult to learn (Surber, 1980).

We were particularly interested in structural mathematical relations because of their likely role in mathematical problem solving. A growing body of evidence suggests that mathematical problem solving may involve a process like structure mapping (Gentner, 1983). Under this hypothesis, the semantic or intuitive representation and the representation of mathematical operations must have structurally similar components. The particular relationships tested in the present study were chosen because there is considerable evidence that children and adults represent analogous semantic relationships about physical systems (e.g., Dixon \& Tuccillo, 2001). For example, children represent the combination of weights with principles such as range (i.e., the answer must be greater than each of the two weights being combined). If children are using this principle in a structure-mapping process, the children should have a representation of a similar relationship for mathematical operations. The RO principle specifies just such an analogous relationship for addition.

Differences in the quality of the representation of the mathematical operations may explain, in part, the performance of children who understand the structure of the problem (i.e., have a good intuitive representation) but do not generate the correct solution operation (e.g., Dixon \& Moore, 1996). There is little question that these children are very familiar with the correct operation. That is, they can recall operation "facts" and execute the appropriate procedures. The problem appears to be connecting the operation with the problem. Deficits in the representation of the operations may explain this difficulty with problem solving.

\section{REFERENCES}

Bassok, M., Chase, V. M., \& Martin, S. A. (1998). Adding apples and oranges: Alignment of semantic and formal knowledge. Cognitive Psychology, 35, 99-134.

CAmpbell, J. I. D. (1999). Division by multiplication. Memory \& Cog nition, 27, 791-802.

Dixon, J. A., \& Moore, C. F. (1996). The developmental role of intuitive principles in choosing mathematical strategies. Developmental Psychology, 32, 241-253.

Dixon, J. A., \& Moore, C. F. (1997). Characterizing the intuitive representation in problem solving: Evidence from evaluating mathematical strategies. Memory \& Cognition, 25, 395-412.

Dixon, J. A., \& TuCCILlo, F. (2001). Generating initial models for reasoning. Journal of Experimental Child Psychology, 78, 178-212.

GEARY, D. C. (1994). Children's mathematical development: Research and practical applications. Washington, DC: American Psychological Association.

GEARY, D. C. (1995). Reflections of evolution and culture in children's cognition: Implications for mathematical development and instruction. American Psychologist, 50, 24-37.

Gentner, D. (1983). Structure-mapping: A theoretical framework for analogy. Cognitive Science, 7, 155-170.

Gentner, D., \& Markman, A. B. (1997). Structure mapping in analogy and similarity. American Psychologist, 52, 45-56.

Hardiman, P. T., Dufresne, R., \& Mestre, J. P. (1989). The relation between problem categorization and problem solving among experts and novices. Memory \& Cognition, 17, 627-638.

Kintsch, W., \& Greeno, J. G. (1985). Understanding and solving word arithmetic problems. Psychological Review, 92, 109-129.

KrUEgER,L. E. (1986). Why $2 \times 2=5$ looks so wrong: On the odd-even rule in product verification. Memory \& Cognition, 14, 141-149.

Krueger, L. E., \& Hallford, E. W. (1984). Why $2+2=5$ looks so wrong: On the odd-even rule in sum verification. Memory \& Cognition, 12, 171-180.

Lemaire, P., \& REDER, L. (1999). What affects strategy selection in arithmetic? The example of parity and five effects on product verification. Memory \& Cognition, 27, 364-382.

LeVin, I. P., Johnson, R. D., \& FARAone, S. V. (1984). Information integration in price-quality tradeoffs: The effect of missing information. Memory \& Cognition, 12, 96-102.

Miller, K., Perlmutter, M., \& Keating, D. (1984). Cognitive arithmetic: Comparison of operations. Journal of Experimental Psychology: Learning, Memory, \& Cognition, 10, 46-60.

Moore, C. F., Dixon, J. A., \& Haines, B. A. (1991). Components of understanding in proportional reasoning: A fuzzy set representation of developmental progression. Child Development, 62, 441-459.

National Council of Teachers of Mathematics (1989). Curriculum and evaluation standards for school mathematics. Reston, VA: Author.

NESHER, P. (1982). Levels of description in the analysis of addition and 
subtraction word problems. In T. P. Carpenter, J. M. Moser, \& T. A. Romberg (Eds.), Addition and subtraction: A cognitive perspective (pp. 25-38). Hillsdale, NJ: Erlbaum.

Novick, L. R., \& Holyoak, K. J. (1991). Mathematical problem solving by analogy. Journal of Experimental Psychology: Learning, Memory, \& Cognition, 17, 398-415.

Reese, C. M., Miller, K. E., Mazzeo, J., \& Dossey, J. A. (1997). NAEP 1996 mathematics report card for the nation and the states. Washington, DC: National Center for Education Statistics.

Rittle-Johnson, B., \& Siegler, R. S. (1998). The relation between conceptual and procedural knowledge in learning mathematics: A review. In C. Donlan (Ed.), The development of mathematical skills: Studies in developmental psychology (pp. 75-110). Hove, U.K.: Taylor \& Francis, Psychology Press.

Ross, B. H., \& KILBANE, M. C. (1997). Effects of principle explanation and superficial similarity on analogical mapping in problem solving. Journal of Experimental Psychology: Learning, Memory, \& Cognition, 23, 427-440.

Schneider, W., Gruber, H., Gold, A., \& Opwis, K. (1993). Chess expertise and memory for chess positions in children and adults. Journal of Experimental Child Psychology, 56, 328-349.

Stevens, S. S. (1974). Perceptual magnitude and its measurement. In E. C. Carterette \& M. P. Friedman (Eds.), Handbook of Perception: Vol. II. Psychophysical judgment and measurement (pp. 361-389). New York: Academic Press.

Surber, C. F. (1980). The development of reversible operations in judgments of ability, effort, and performance. Child Development, $\mathbf{5 1}$, 1018-1029.

\section{NOTES}

1. For each operation, an additional subdesign was constructed in which we independently manipulated (1) amount wrong, the average absolute distance of the presented answers to the correct answers, and
(2) maximum distance, the largest deviation from the correct answer of any answer presented in the problem set. Maximum distance was manipulated because participants' ratings may be disproportionately affected by answers that are extremely wrong. In creating the problem sets for the main design, we kept the maximum distance as small as possible. However, in the maximum distance subdesign, we strongly manipulated the maximum distance independently of the average distance to assess its possible effect. The results showed that the participants did not rate problem sets with larger maximum distances as worse examples of the operations.

2. Details on the levels of amount wrong used in each subdesign are available on line (http://faculty.wm.edu/jadixo/amtwrng.pdf). Pilot testing and the results of Experiments 1 and 2 showed that the levels of amount wrong used had linear effects on the participants' ratings and were unrelated to whether or not principle violations were detected.

3 . Trials that violate the Mono principle are also necessarily nonlinear. Therefore, comparison trials with the same degree of nonlinearity and amount wrong were constructed, as well as comparison trials that were linear and had the same amount wrong. There was no significant difference between these comparison problems; we report the latter set. The DE principle is also violated by trials that violate Mono. This relationship is unavoidable.

4. The interaction between principle violation and amount wrong would be of potential interest for identifying how participants subjectively combine these types of information.

5. The induction hypothesis also predicts that less complex principles (those based on fewer comparisons) should be induced first. RO, $\mathrm{DE}$, Mono, and Slopes require comparing relationships among one, two, three, and four problems, respectively, and therefore, should be induced in that order. However, contrary to this prediction, the data show that Mono is present without RO or DE.

6. A second sample of college students was explicitly told that the numbers were all positive integers. Their results were identical to those reported here. 


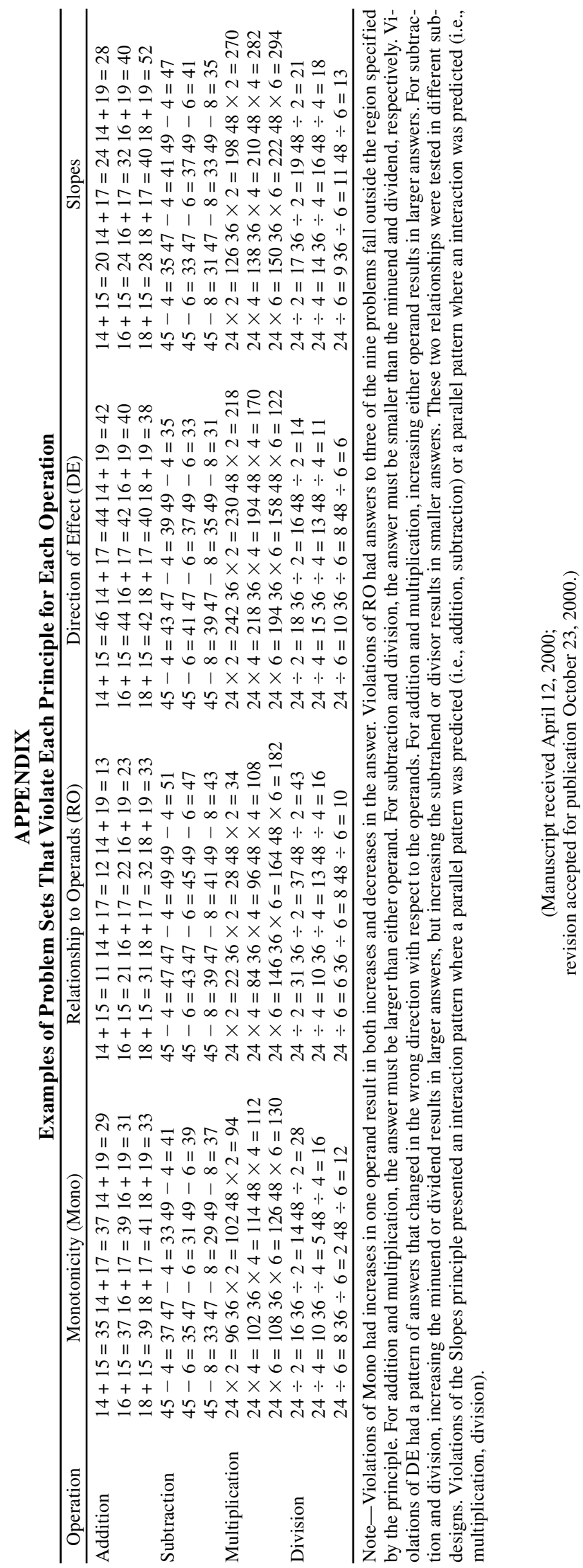

